\title{
Epidemiology of NMOSD in Catalonia: Influence of the new 2015 criteria in incidence and prevalence estimates
}

\author{
Maria Sepúlveda, Marta Aldea, Domingo Escudero, Sara Llufriu, Georgina Arrambide, Susana \\ Otero-Romero, J Sastre-Garriga, Lucía Romero-Pinel, Sergio Martínez-Yélamos, N Sola-Valls, \\ Thais Armangué, Javier Sotoca, Antonio Escartín, René Robles-Cedeño, Lluís Ramió-Torrentà, \\ Silvia Presas-Rodríguez, Cristina Ramo-Tello, Elvira Munteis, Raúl Pelayo, Laura Gubieras, \\ Luis Brieva, Nicolau Ortiz, Mariona Hervás, Maria Alba Mañé-Martínez, Antonio Cano, Emili \\ Vela, Mar Tintoré, Yolanda Blanco, Xavier Montalban, Francesc Graus and Albert Saiz
}

\section{Abstract}

Background: Population-based studies on neuromyelitis optica spectrum disorders (NMOSD) are limited, and it is unclear whether the rates have changed with the implementation of the new 2015 criteria.

Objectives: To estimate the incidence and prevalence of NMOSD in Catalonia (Spain), using both the 2006 and the 2015 criteria.

Methods: In this clinic-based retrospective study, patients diagnosed with NMOSD between 2006 and 2015 were identified using multiple sources, including direct contact to all Catalan hospitals, identification of cases through the Catalan Health Surveillance System, and registry of antibodies to aquaporin-4 (AQP4-IgG) and myelin oligodendrocyte glycoprotein (MOG-IgG) in a reference laboratory. The incidence rate was calculated for the period 1 January 2006-1 January 2016 and prevalence for the date 1 January 2016.

Results: We identified 74 patients (by the 2015 criteria). Most patients were Caucasian (81\%), and female (76\%) with a median age at disease onset of 42 years (range, 10-76 years). In total, 54 (73\%) patients were positive for AQP4-IgG, 11 (15\%) double-seronegative, and 9 (12\%) MOG-IgG-positive. Rates of incidence and prevalence $(0.63 / 1,000,000$ person-years and $0.89 / 100,000$, respectively) were 1.5 -fold higher than those reported by the 2006 criteria. Lowest rates were seen in children and elder people and highest in women and middle-aged people (40-59years). The female predominance was lost in incident AQP4IgG-seronegative children and AQP4-IgG-positive elder people. MOG-IgG and double-seronegativity contributed similarly but did not influence the long-term outcome.

Conclusion: The new criteria increase the estimates, but NMOSD remains as a rare disease. The differences in age- and sex-specific estimates highlight the importance of the serologic classification.

Keywords: Neuromyelitis optica spectrum disorders, prevalence, incidence, AQP4-antibodies, MOGantibodies

Date received: 21 June 2017; revised: 4 September 2017; accepted: 11 September 2017

\section{Introduction}

Neuromyelitis optica (NMO) is an inflammatory demyelinating central nervous system disease characterized by frequent relapses mainly involving the optic nerves and the spinal cord. The discovery of antibodies to aquaporin-4 (AQP4-IgG) in 2004 as a specific biomarker of NMO led to the proposal of new clinical criteria in $2006^{1}$ and a modification of these criteria in 2015 which included several syndromes under the term of NMO spectrum disorders (NMOSD). ${ }^{2}$
Multiple Sclerosis Journal

$1-9$

DOI: $10.1177 /$

1352458517735191

(C) The Author(s), 2017. Reprints and permissions: http://www.sagepub.co.uk/ journalsPermissions.nav
Correspondence to: A Saiz

Center of Neuroimmunology, Laboratory of Advanced Imaging in Neuroimmunological Diseases, Hospital Clinic of Barcelona and Institut d'Investigacions Biomediques August Pi i Sunyer (IDIBAPS), University of Barcelona, Villarroel 170, 08036 Barcelona, Spain. asaiz@clinic.ub.es

Maria Sepúlveda Domingo Escudero Sara Llufriu N Sola-Valls Yolanda Blanco Francesc Graus Albert Saiz Center of Neuroimmunology, Laboratory of Advanced Imaging in Neuroimmunological Diseases, Hospital Clinic of Barcelona and Institut d'Investigacions Biomediques August Pi i Sunyer (IDIBAPS), University of Barcelona, Barcelona, Spain

Marta Aldea Department of Preventive Medicine and Epidemiology, Hospital Clinic of Barcelona, University of Barcelona, Barcelona, Spain

Georgina Arrambide J Sastre-Garriga Mar Tintoré Servei de NeurologiaNeuroimmunologia, Centre d'Esclerosi Múltiple de Catalunya (Cemcat), Vall d'Hebron Institut de Recerca, Hospital Universitari Vall d'Hebron, Universitat Autònoma de Barcelona, Barcelona, Spain 
Susana Otero-Romero Servei de NeurologiaNeuroimmunologia, Centre d'Esclerosi Múltiple de Catalunya (Cemcat),

Vall d'Hebron Institut de Recerca, Hospital Universitari Vall d'Hebron, Universitat Autònoma de Barcelona, Barcelona, Spain Department of Preventive Medicine and Epidemiology, Hospital Universitari Vall d'Hebron, Universitat Autònoma de Barcelona, Barcelona, Spain

Lucía Romero-Pinel Sergio Martínez-Yélamos Multiple Sclerosis Unit, Department of Neurology, Hospital Universitari de Bellvitge, Barcelona, Spain

Thais Armangué

Center of Neuroimmunology,

Laboratory of

Advanced Imaging in

Neuroimmunological

Diseases, Hospital

Clinic of Barcelona and

Institut d'Investigacions

Biomediques August

$\mathrm{Pi}$ i Sunyer (IDIBAPS),

University of Barcelona,

Barcelona, Spain/Pediatric

Neuroimmunology and

Neuroinfections Unit,

Hospital Sant Joan de Déu

Barcelona, University of

Barcelona, Spain/Centre

for Biomedical Network

Research on Rare Diseases

(CIBERER), Madrid, Spain

Javier Sotoca

Antonio Escartín

Department of Neurology,

Hospital de la Santa Creu

Sant Pau, Barcelona, Spain

René Robles-Cedeño

Lluís Ramió-Torrentà

Neuroimmunology and

Multiple Sclerosis Unit,

Neurology Department,

Doctor Josep Trueta

University Hospital, Girona,

Spain/ Santa Caterina

Hospital, Girona, Spain

Biomedical Research

Institute (IDIBGI), Girona,

Spain/Medical Sciences

Department, University of

Girona, Girona, Spain

Silvia Presas-Rodríguez

Cristina Ramo-Tello

Multiple Sclerosis Unit,

Neuroscience Department,

Hospital Universitari

Germans Trias i Pujol,

Barcelona, Spain

Elvira Munteis

Multiple Sclerosis Unit,

Neurology Department, Parc

de Salut Mar, Barcelona,

Spain

Raúl Pelayo

Institut Guttmann,

Barcelona, Spain
NMOSD is considered to be a rare disorder associated with poor prognosis and different frequencies depending on the ethnicity. ${ }^{3}$ Population-based studies on NMOSD are infrequent and heterogeneous. Most studies were based on older criteria, ${ }^{4-6}$ and some of them did not describe the methods of AQP4-IgG detection. ${ }^{7,8}$ Whether the implementation of the 2015 criteria has changed the rates of incidence/prevalence is presently unknown, and the comparison with the 2006 criteria in the same population is the most accurate way to answer this question. In addition, recent studies have shown that antibodies to myelin oligodendrocyte glycoprotein (MOG-IgG) may occur in some patients who are AQP4-IgG seronegative, ${ }^{9}$ but their contribution to the burden of the disease remains to be elucidated.

To address these issues, we conducted an epidemiological study to estimate the incidence, and prevalence of NMOSD in Catalonia (Spain), based on both the 2006 and the 2015 criteria.

\section{Methods}

\section{Setting}

Catalonia is a geographically and administratively defined area located in North East Spain with a total extension of $31,895 \mathrm{~km}^{2}$. The total population according to the 1 January 2016 census was 7,522,596 inhabitants with a clear predominance of Caucasian ethnicity $(82.81 \%$ born in Spain, $4.43 \%$ in the rest of Europe, $6.73 \%$ in Latin America, 3.87\% in Africa, and $2.00 \%$ in Asia). Health care in Catalonia is provided by a consolidated open access public healthcare system comprising 67 tertiary and local hospitals and a network of adult and pediatric neurologists specialized in demyelinating diseases.

\section{Case ascertainment}

In this clinic-based multicenter retrospective study, patients diagnosed with NMOSD between 2006 and 2015 according to the 2015 criteria were identified using multiple sources: (1) notification of the study to the Catalan Society of Neurology, the Catalan Neuropediatrics Association, and the Catalan members of the Spanish NMO Study Group ${ }^{9}$ to report known cases under the care of their members; (2) direct contact with all hospitals with multiple sclerosis units and 64 of the 67 Catalan hospitals (covering the $99.6 \%$ of the Catalan population; neurologic patients from the 3 remaining hospitals are referred to identified reference centers); (3) identification of all residents in Catalonia who during the study time period appeared in the Catalan Health Surveillance System (CHSS; a regional epidemiology register that includes the hospitalization diagnoses since 1990, and primary care visits, emergency room consultations, and specialized outpatients visits among others since 2011; Supplementary Material) with a diagnosis of NMOSD (World Health Organization (WHO) ICD-9 code: 341.0); and (4) AQP4-IgG testing laboratory registry (2968 samples analyzed for suspected NMOSD between November 2005 and December 2015, 40\% of them from Catalan cases, Hospital Clinic of Barcelona)

\section{Data collection}

Epidemiological data, including demographic, clinical, cerebrospinal fluid (CSF; cell count, protein levels, and oligoclonal bands), magnetic resonance imaging (MRI) findings (brain MRI classified as normal and abnormal with or without Paty's or Barkhof's criteria, and number and extension of spinal cord lesions), treatment and outcome, were obtained from medical records and information collected from referring neurologists through a structured questionnaire designed for NMOSD as reported. ${ }^{9}$ In total, $71(96 \%)$ of 74 serum samples were tested for AQP4-IgG by cell-based assay, 2 by immunohistochemistry, ${ }^{10}$ and 1 by ELISA. In addition, 60/74 (81\%) patients were also tested for MOG-IgG by cell-based assay. ${ }^{9}$ Fulfillment of the $2006^{1}$ and $2015^{2}$ criteria was revised by two neurologists (M.S. and A.S.), and if needed, additional clinical or MRI information and/or serum samples was obtained from the referring physicians.

This study was approved by the Ethics Committee of the Hospital Clinic of Barcelona. All participants or next of kin (parents of children cases) when appropriate (blood samples) gave written consent.

\section{Case definition and estimation of incidence and prevalence}

The prevalence calculated for the date of 1 January 2016 was the number of prevalent NMOSD cases per 100,000 inhabitants. Cases were considered prevalent if they had a confirmed NMOSD diagnosis according to 2015 criteria and were alive and resident in the study region on the established prevalence data. The incidence rate was the number of new NMOSD cases for the period 1 January 2006-1 January 2016 divided by the total number of people-years at risk (estimated by summing up the mid-year census population of each year ${ }^{11}$ ) and was reported per $1,000,000$ peopleyears. Incident cases were defined as confirmed NMOSD patients (criteria 2015) who had the onset of 
symptoms compatible with a clinical core episode of NMOSD during the study time period (optic neuritis, acute myelitis, area postrema syndrome, acute brainstem syndrome, symptomatic narcolepsy or acute diencephalic clinical syndrome, and symptomatic cerebral syndrome) and were positive for AQP4-IgG or negative for AQP4-IgG but with two clinically documented core episodes with spatial dissemination and additional MRI requirements. ${ }^{2}$ Prevalence and incidence rates were also assessed according to 2006 criteria: optic neuritis and myelitis along with two of the following three supportive criteria: (1) longitudinally extensive transverse myelitis ( $\geq 3$ vertebral segments in length), (2) brain MRI with normal findings or with findings not consistent with multiple sclerosis, and (3) AQP4-IgG seropositivity. ${ }^{1}$

Population estimates for Catalonia were obtained from the website of the Statistics Institute of Catalonia. ${ }^{12}$ Exact date of symptom onset was available for all cases, and the medical record review of the patients was continued until 1 January 2017 to diagnose incident cases that had a delayed presentation. As AQP4-IgG determination was not available until November 2005, the presence of this antibody was determined on stored sera in patients who had been diagnosed with NMO before 2006.

\section{Statistical methods}

Crude and sex- and age-specific prevalence and incidence rates were calculated using four age groups $(0-18,19-39,40-59$, and $\geq 60$ years). In total, $95 \%$ confidence intervals (CIs) were calculated for all prevalence and incidence rate estimates. Agestandardized prevalence and incidence rates were calculated by the direct method using the WHO World Standard Population. Quantitative variables were described using mean \pm standard deviation (SD) or median and range. The values of $p \leq 0.05$ were considered significant. Analyses were performed using SPSS statistical software, v. 19.0.

\section{Results}

We identified a total of 74 NMOSD patients confirmed by the 2015 criteria. The identification of cases by each method is detailed in Supplementary Material. Demographical and clinical characteristics of these patients are summarized in Table 1. NMOSD patients were mostly White $(81 \%)$, followed by Hispanic ethnicity $(11 \%)$. The female:male ratio was 3.1:1 and the median age at onset 42 years (range, 10-76years). In total, 54 (73\%) patients were AQP4-IgG-seropositive, $11(15 \%)$ double-seronegative, and 9 (12\%)
MOG-IgG-positive. None of the AQP4-IgGseropositive patients were positive for MOG-IgG.

No differences were noted among patients according to serostatus except for lower final Expanded Disability Status Scale (EDSS) in MOG-IgGpositive patients $(p=0.030$; Supplementary Table S1). An analysis of patients with at least 10 years of disease duration showed that 14/32 (44\%) had an EDSS $\leq 3.0$. When these patients were compared to those with EDSS $>3.0$, we found that the serologic distribution (AQP4-IgG, MOG-IgG, and double-seronegative) in both groups was similar, and the only difference was the higher frequency of optic neuritis onset attack $(p=0.047)$ and relapse rate $(p=0.009)$ in patients with EDSS $>3.0$ (Supplementary Table S2). The frequency distribution of cases according to the serologic status, by gender and age, is shown in Figure 1.

\section{Incidence and prevalence}

There were 47 incident cases according to 2015 criteria over the period 2006-2016, giving a crude incidence rate of 0.63 (95\% CI: $0.45-0.81$ ) per million. Prior to the prevalence date, three patients died and four were not anymore residents in Catalonia leaving 67 prevalent cases and a crude point prevalence of 0.89 (95\% CI: $0.87-0.91$ ) per 100,000 persons (Table 2). Incidence and prevalence rates stratified by groups of age and sex are shown in Table 3. Overall annual incidence and by sex is shown in Supplementary Figure S1. The estimates of incidence and prevalence with the 2015 criteria were 1.5 -fold higher than that reported by the 2006 criteria (Table 2). Agestandardized incidence rate to the WHO population was 0.64 (95\% CI: 0.45-0.82) per million and the prevalence of 0.95 (95\% CI: $0.76-1.14)$ per 100,000.

The expected number of cases in the Spanish population based on age-standardized incidence was 30 (95\% CI: 21-38) and that for prevalent cases 441 (95\% CI: 352-530).

\section{Seroincidence and seroprevalence according to serologic status}

The estimates for AQP4-IgG-positive, double-seronegative, and MOG-IgG-positive, stratified by age and sex are shown in Table 3. Overall AQP4-IgG-positive serologic incidence increased with the patient's age from 0.15 in children to 0.62 per million in middleaged people. MOG-IgG-positive serologic incidence followed a similar increase pattern with a maximum peak of 0.14 per million in middle-aged people, while
Laura Gubieras

Multiple Sclerosis Unit,

Neurology Department,

Hospital de Sant Joan Despi

Moisès Broggi, Barcelona,

Spain

Luis Brieva

Multiple Sclerosis Unit,

Neurology Department,

Hospital Universitari Arnau

de Vilanova, Lleida, Spain

Nicolau Ortiz

Neurology Department,

Hospital Universitari Sant Joan de Reus, Tarragona,

Spain

Mariona Hervás

Multiple Sclerosis Unit,

Neurology Department,

Hospital Parc Taulí de

Sabadell, Barcelona, Spain

Maria Alba Mañé-Martínez

Neurology Department,

Hospital Universitari de

Tarragona Joan XXIII,

Tarragona, Spain

Antonio Cano

Neurology Department,

Hospital de Mataró,

Barcelona, Spain

Emili Vela

Unit of Information and Knowledge, Catalan Health Service, Barcelona, Spain

Xavier Montalban

Servei de Neurologia-

Neuroimmunologia, Centre

d'Esclerosi Múltiple de

Catalunya (Cemcat), Vall

d'Hebron Institut de Recerca,

Hospital Universitari Vall

d'Hebron, Universitat

Autònoma de Barcelona,

Barcelona, Spain; Division

of Neurology, St. Michael's

Hospital, University of

Toronto, Toronto, ON,

Canada 
Table 1. Demographic and clinical characteristics of patients with NMOSD.

\begin{tabular}{|c|c|}
\hline & $\begin{array}{l}\text { Patients } \\
(n=74)\end{array}$ \\
\hline Female sex, $n(\%)$ & $56(76)$ \\
\hline Ratio female:male & $3.1: 1$ \\
\hline \multicolumn{2}{|l|}{ Ethnic, $n(\%)$} \\
\hline White & $60(81)$ \\
\hline Hispanic & $8(11)$ \\
\hline Black & $2(3)$ \\
\hline Asian & $2(3)$ \\
\hline Arab & $2(3)$ \\
\hline \multicolumn{2}{|l|}{ Serologic status, $n(\%)$} \\
\hline AQP4-IgG & $54(73)$ \\
\hline MOG-IgG & $9(12)$ \\
\hline Double-seronegative & $11(15)$ \\
\hline Age at onset (years), median (range) & $42(10-76)$ \\
\hline \multicolumn{2}{|l|}{ Onset attack type, $n(\%)$} \\
\hline Optic neuritis & $28(38)$ \\
\hline Myelitis & $30(41)$ \\
\hline Optic neuritis + myelitis & $10(14)$ \\
\hline Area postrema syndrome ${ }^{a}$ & $3(4)$ \\
\hline Acute brainstem syndrome ${ }^{b}$ & $3(4)$ \\
\hline \multicolumn{2}{|l|}{ Brain MRI, $n(\%)$} \\
\hline Normal & $29(39)$ \\
\hline Nonspecific WM lesions & $33(45)$ \\
\hline Barkhof's criteria & $6(8)$ \\
\hline Other & $6(8)$ \\
\hline \multicolumn{2}{|l|}{ Spinal MRI, $n(\%)$} \\
\hline LETM & $61(82)$ \\
\hline No. of vertebral segment, mean (SD) & $6.7(5.1)$ \\
\hline Short myelitis & $5(7)$ \\
\hline Coexisting autoimmune disease, $n(\%)$ & $20(27)$ \\
\hline Chronic treatment, $n(\%)$ & $61(82)$ \\
\hline Relapsing forms, $n(\%)$ & $64(87)$ \\
\hline Annualized relapse rate, mean (SD) & $0.9(1.5)$ \\
\hline Time to last follow-up (years), mean (SD) & $9.7(7.8)$ \\
\hline Last EDSS score, median (range) & $3.5(0-10.0)$ \\
\hline Patients who died, $n(\%)$ & $4(5)$ \\
\hline \multicolumn{2}{|c|}{$\begin{array}{l}\text { NMOSD: neuromyelitis optica spectrum disorders; SD: } \\
\text { standard deviation; AQP4-IgG: antibodies to aquaporin-4; } \\
\text { EDSS: Expanded Disability Status Scale; MRI: magnetic } \\
\text { resonance imaging; MOG-IgG: antibodies to myelin } \\
\text { oligodendrocyte glycoprotein; LETM: longitudinally extensive } \\
\text { transverse myelitis; WM: white matter. } \\
\text { aPlus concomitant myelitis in one case. } \\
\text { bPlus optic neuritis in one case. } \\
\text { 'Midbrain lesion with periependymal dorsal involvement (two } \\
\text { cases), dorsal medulla lesion (area postrema: three cases), or } \\
\text { with periependymal involvement (1 case). }\end{array}$} \\
\hline
\end{tabular}

the peak for double-seronegativity was identified in children 0.15 and middle-aged people 0.14 per million. The female predominance was lost in AQP4-IgG seronegative incident children (MOG-IgG and double-seronegative) and AQP4-IgG positive elder people (Table 3 ). The seroprevalence results are shown in Table 3 and Figure 1.

\section{Discussion}

This clinic-based study of NMOSD provides several important findings: (1) the implementation of the 2015 criteria has increased the rates of incidence and prevalence. However, the rates remain low in a predominant Caucasian European population; (2) lowest rates are seen in children ( $<18$ years) and elder people ( $>60$ years), and the maximum peak in middle-aged people (40-59years); (3) the overall rates in females patients exceed those of male patients by a factor of approximately 3 , but the female predominance is lost in AQP4-IgG-negative children and AQP4-IgGseropositive elder people; (4) MOG-IgG and doubleseronegativity contribute with similar rates; and (5) some NMOSD patients have a favorable outcome that does not seem to be influenced by the serologic status.

The finding of estimates of incidence and prevalence 1.5 -fold higher with the 2015 criteria compared to those of the 2006 criteria is not unexpected. The 2015 criteria allow the diagnosis of NMOSD in patients AQP4-IgG-positive who had a single episode or limited forms of the disease, whereas the criteria are more stringent for patients AQP4-IgG-negative, requiring at least two attacks with dissemination in space and additional MRI findings. ${ }^{2}$ The fact that the application of the new criteria identified 18 additional prevalent AQP4-IgG-positive cases, and 5 AQP4IgG-negative cases, reinforces the importance of including AQP4-IgG-negative NMOSD in epidemiological studies using uniform and recognized case definitions. $^{6}$

Our estimate of prevalence is comparable to that of a recent study in Australia and New Zealand $(0.70 / 100,000),{ }^{13}$ which until now was the only study based on the 2015 criteria (Supplementary Table S3). Although our incidence rate was almost twofold higher, the prevalence:incidence ratio, as indicator of disease duration, was similar (14:1 vs 19:1, respectively), and comparable to that reported in Denmark ${ }^{5}$ and Martinique. ${ }^{6}$ It is noteworthy that the Australia and New Zealand study differed in the high rate of patients of Asian ancestry (12\%) and higher prevalence $(1.23 / 100,000)$ in that population. ${ }^{13}$ Other population-based studies have shown ethnics differences, with the highest rates in patients of African origin (prevalence up to 2.5 times higher than in Caucasian population). ${ }^{6}$ Our rates of 
prevalence were comparable to other studies that included limited AQP4-IgG-positive forms ${ }^{8}$ but lower than those found in a study from Olmsted County $(3.9 / 100,000)^{6}$ and Denmark $(4.4 / 100,000) .{ }^{5}$ However, only the latter reported a significant higher incidence rate $^{6}$ (Supplementary Table S3).

In our study, the incidence and prevalence in females exceeded those of male patients by a factor of 3 , confirming the known female predominance for people diagnosed with NMOSD (Supplementary Table S3). Previous population-based studies did not assess the results by age-sex strata. We found the lowest incident rates in children and elder people, who overall accounted for $20 \%$ of cases, and the highest rates for the middle-aged population. Except for differences in the incident peak age, this distribution is not different from that described in other autoimmune inflammatory diseases such as multiple sclerosis or systemic lupus erythematosus..$^{14,15}$ Moreover, the female predominance was not observed in incident patients aged $>60$ years. When examining differences according to the serologic status, it was due to an excess of incident male in AQP4-IgG-positive elder patients.

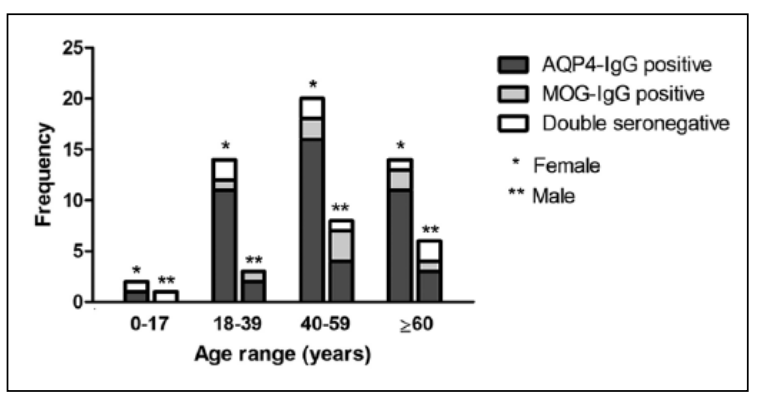

Figure 1. Distribution of NMOSD cases according to gender, age, and serologic status.
Similarly, the female predominance observed in incident children, was lost in AQP4-IgG-seronegative (MOG-IgG and double-seronegative). In contrast, the female predominance remained across the four age groups in the analysis of overall prevalence. In fact, the serologic prevalence of AQP4-IgG-positive cases with lower rates in male children is in agreement with findings from a pediatric NMOSD cohort ${ }^{16}$ and laboratory-based study. ${ }^{17}$ However, our study ruled out the overrepresentation of MOG-IgG in seronegative pediatric NMOSD patients because only one of the three seronegative incident cases was MOG-IgG-positive. Overall, these findings emphasize the importance of the serologic classification of the new criteria.

The association of MOG-IgG with NMOSD has been increasingly recognized, ${ }^{9}$ but there are no populationbased studies that to date have analyzed its contribution to the disease. Our study shows similar rates of serologic incidence for MOG-IgG and double-seronegativity. Incident MOG-IgG cases appeared across the four age groups, with a mild female predominance except in children. Although the overall outcome in terms of disability was better for MOG-IgG-positive patients, the analysis of patients with at least 10 years of disease duration showed that MOG-IgG positive cases were distributed equally between low and high EDSS groups $(\leq 3.0$ or $>3.0)$. The presentation with optic neuritis attack at onset and a higher relapse rate, but not the serologic status, were the only factors associated with a worst outcome (EDSS $>3.0)$. Thus, our study confirms previous clinic-based data that a "benign" or favorable outcome appears to occur in some NMOSD patients, ${ }^{18}$ and it seems to be independent of the serologic status. The identification of these patients is important for the search of prognostic biomarkers in NMOSD.

Table 2. Estimates of prevalence and incidence of NMOSD according to 2015 and 2006 criteria.

\begin{tabular}{|c|c|c|c|c|}
\hline \multirow[t]{2}{*}{ NMOSD } & \multicolumn{2}{|c|}{2015 NMOSD criteria } & \multicolumn{2}{|c|}{2006 NMO criteria } \\
\hline & $\begin{array}{l}\text { Prevalent cases, } \\
2016\end{array}$ & $\begin{array}{l}\text { Prevalence/100,000 } \\
(95 \% \mathrm{CI})\end{array}$ & $\begin{array}{l}\text { Prevalent } \\
\text { cases, } 2016\end{array}$ & $\begin{array}{l}\text { Prevalence/ } \\
100,000(95 \% \mathrm{CI})\end{array}$ \\
\hline Overall & 67 & $0.89(0.87-0.91)$ & 44 & $0.58(0.57-0.60)$ \\
\hline Female & 50 & $1.31(1.27-0.34)$ & 32 & $0.84(0.81-0.87)$ \\
\hline \multirow[t]{2}{*}{ Male } & 17 & $0.46(0.44-0.48)$ & 12 & $0.32(0.31-0.34)$ \\
\hline & $\begin{array}{l}\text { Incident cases, } \\
\text { 2006-2016 }\end{array}$ & $\begin{array}{l}\text { Incidence } / 1,000,000 \\
\text { person-years }(95 \% \mathrm{CI})\end{array}$ & $\begin{array}{l}\text { Incident cases, } \\
2006-2016\end{array}$ & $\begin{array}{l}\text { Incidence } / 1,000,000 \\
\text { person-years }(95 \% \mathrm{CI})\end{array}$ \\
\hline Overall & 47 & $0.63(0.45-0.81)$ & 30 & $0.40(0.26-0.55)$ \\
\hline Female & 34 & $0.90(0.60-1.21)$ & 22 & $0.58(0.34-0.83)$ \\
\hline Male & 13 & $0.35(0.16-0.54)$ & 8 & $0.22(0.07-0.37)$ \\
\hline
\end{tabular}




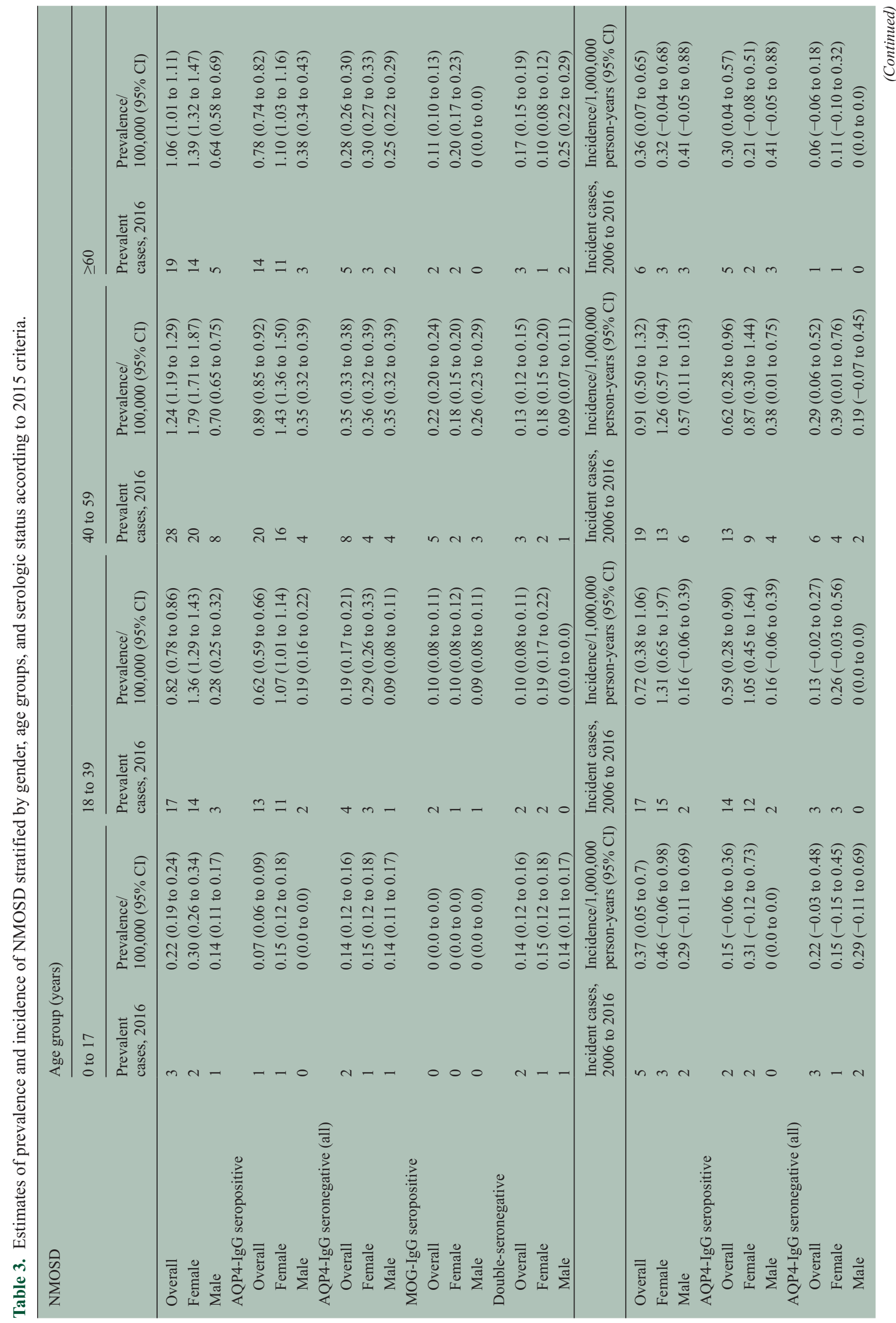




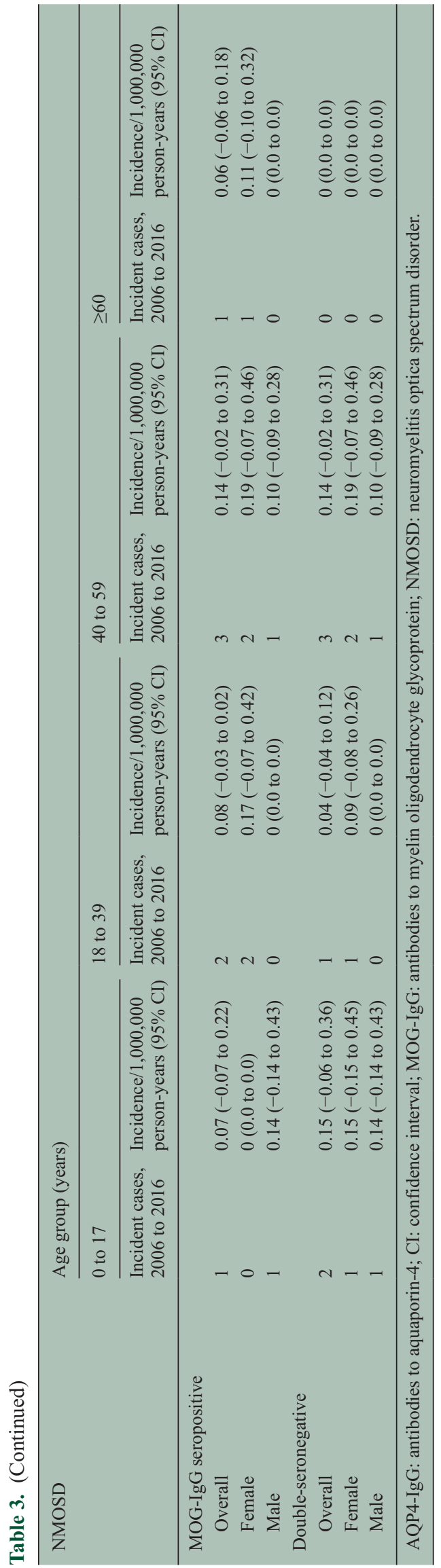

The strengths of this study include the use of multiple source of case ascertainment. These were the network of neurologists that we set up to describe the features of NMOSD in Spain, ${ }^{9}$ and pediatric neurologists involved in a national prospective study of pediatric patients with a first demyelinating episode, coupled with a reference laboratory of neuroimmunology, and the registries of the CHSS. A limitation was that the CHSS registries started in 2011 the inclusion of primary care and specialized outpatients visits, ${ }^{19}$ and only those patients coded as NMO and Devic disease were revised. It is likely, however, that the direct contact with all Catalan hospitals may have overcome in part this limitation. Additionally, we cannot rule out an underrepresentation of cases prior to 2005 when AQP4-IgG testing was available and a selection bias toward more recently active cases. However, the observed stable rate of incidence over the period of study seems to rule out the latter. Nevertheless, the large number of samples tested suggests that antibody testing is frequently performed in unselected cases of inflammatory demyelinating diseases.

In conclusion, our study shows an increase in the incidence and prevalence estimates with the new criteria, although NMOSD remains as a rare disease in a predominant Caucasian population. The serologic classification is important because the rates differ according to the sex and age distribution. In addition, the study provides standardized results and subgroupspecific estimates using uniform definition, an important fact to enhance comparability of studies from different populations.

\section{Declaration of Conflicting Interests}

The author(s) declared the following potential conflicts of interest with respect to the research, authorship, and/or publication of this article: M.S. received speaker honoraria from Genzyme and Novartis. S.L. received speaker honoraria from Biogen-Idec, Novartis, Teva, Genzyme, and Merck and research support from a Juan Rodes grant from the Instituto de Salud Carlos III (JR14/00016) and the Spanish Government (PI15/00587). G.A. has received compensation for consulting services from Biogen-Idec, research support from Novartis, and speaking honoraria from Sanofi-Aventis. S.O.-R. has received compensation for consulting services from Biogen-Idec and Genzyme and research support from Novartis. J.S.-G. has received in the last 12 months compensation for participating on advisory boards; speaking honoraria and travel expenses for scientific meetings; consulting services; or research support from Novartis, Excemed, Merck, Teva, Celgene, and Genzyme. L.R.-P. received research support; funding 
for travel and congress expenses; and honoraria from speaking engagements and scientific advisory board from Biogen-Idec, Teva Pharmaceutical Industries Ltd, Sanofi-Aventis, Novartis, Bayer HealthCare Pharmaceuticals, and Merck Serono. S.M.-Y. has received honoraria compensation for participating in advisory boards; acting as consultant; and for speaking activities from Biogen-Idec, Teva, Sanofi-Aventis, Merck Serono, Novartis, and Farmacéutica Bayer Schering. N.S.-V. receives funding from the Spanish Government (Instituto de Salud Carlos III, Spain and Fondo Europeo de Desarrollo Regional; FEDER, FI16/00251) and a Predoctoral Grant for Health Research. R.R.-C. had received speaker honoraria from Bayer, Biogen, and Novartis. L.R.-T. has received compensation for consulting services and speaking honoraria from Biogen-Idec, Novartis, Bayer, Merck Serono, Genzyme, Teva Pharmaceutical Industries Ltd, and Almirall. C.R.-T. received travel funding from Biogen and Novartis and received research support from Biogen, Genzyme, Novartis, and Almirall. E.M. has received compensation for consulting services and speaking honoraria from Biogen-Idec, Novartis, Merck Serono, Genzyme, and Almirall. L.B. has received speaking honoraria from Biogen-Idec, Novartis, Merck Serono, Genzyme, and Almirall. N.O. has received support for travel and congress expenses from Sanofi-Aventis and Novartis. M.A.M.-M. has received research support and funding for travel and congress expenses from BiogenIdec, Teva Pharmaceutical Industries, Sanofi-Aventis, Merck Serono, Novartis, and Bayer Schering Pharma. M.T. has received compensation for consulting services and speaking honoraria from Bayer Schering Pharma, Merck Serono, Biogen-Idec, Teva Pharmaceuticals, Sanofi-Aventis, Novartis, Almirall, Genzyme, and Roche. Y.B. received speaking honoraria from Biogen, Novartis, and Genzyme. X.M. has received speaking honoraria and travel expenses for participation in scientific meetings; has been a steering committee member of clinical trials; or participated in advisory boards of clinical trials in the past with Actelion, Almirall, Bayer, Biogen, Celgene, Genzyme, Hoffmann-La Roche, Novartis, Oryzon Genomics, Sanofi-Genzyme, and Teva Pharmaceutical. A.S. received compensation for consulting services and speaker honoraria from Bayer Schering, Merck Serono, Biogen-Idec, SanofiAventis, Teva, and Novartis. M.A., D.E., T.A., J.S., A.E., S.P.-R., R.P., L.G., M.H., A.C., E.V., and F.G. report no disclosures.

\section{Funding}

The author(s) disclosed receipt of the following financial support for the research, authorship, and/or publication of this article: This work was funded by a grant from La Marató de TV3 (20141830; F.G., M.T., and A.S.), Proyecto de Investigacion en Salud (FIS 2015. PI15/00587, S.L. and A.S.), and Red Española de Esclerosis Múltiple (RD16/0015/0002) integrated in the Plan Estatal de Investigación Científica y Técnica de Innovación $\mathrm{I}+\mathrm{D}+\mathrm{I}$ and co-funded by the Instituto de Salud Carlos III-Subdirección General de Evaluación and the Fondo Europeo de Desarrollo Regional (FEDER, "Otra manera de hacer Europa"). S.L. is recipient of a Juan Rodes grant from the Instituto de Salud Carlos III (JR14/00016). M.S. receives funding from the Generalitat de Catalunya (SLT002/16/00354). T.A. has received funding from Instituto Carlos III, Madrid, Spain (CM14/00081 and CV15/00021).

\section{References}

1. Wingerchuk DM, Lennon VA, Pittock SJ, et al. Revised diagnostic criteria for neuromyelitis optica. Neurology 2006; 66: 1485-1489.

2. Wingerchuk DM, Banwell B, Bennett JL, et al. International consensus diagnostic criteria for neuromyelitis optica spectrum disorders. Neurology 2015; 85: 177-179.

3. Pandit L, Asgari N, Apiwattanakul M, et al. Demographic and clinical features of neuromyelitis optica: A review. Mult Scler 2015; 21: 845-853.

4. Cabrera-Gomez JA, Kurtzke JF, Gonzalez-Quevedo A, et al. An epidemiological study of neuromyelitis optica in Cuba. J Neurol 2009; 256: 35-44.

5. Asgari N, Lillevang ST, Skejoe HP, et al. A population-based study of neuromyelitis optica in Caucasians. Neurology 2011; 76: 1589-1595.

6. Flanagan EP, Cabre P, Weinshenker BG, et al. Epidemiology of aquaporin-4 autoimmunity and neuromyelitis optica spectrum. Ann Neurol 2016; 79 : 775-783.

7. Cossburn M, Tackley G, Baker K, et al. The prevalence of neuromyelitis optica in South East Wales. Eur J Neurol 2012; 19: 655-659.

8. Jacob A, Panicker J, Lythgoe D, et al. The epidemiology of neuromyelitis optica amongst adults in the Merseyside county of United Kingdom. $J$ Neurol 2013; 260: 2134-2137.

9. Sepúlveda M, Armangué T, Sola-Valls N, et al. Neuromyelitis optica spectrum disorders: Comparison according to the phenotype and serostatus. Neurol Neuroimmunol Neuroinflamm 2016; 3: e225.

10. Höftberger R, Sabater L, Marignier R, et al. An optimized immunohistochemistry technique improves 
NMO-IgG detection: Study comparison with cellbased assays. PLoS ONE 2013; 8: e79083.

11. Vandenbroucke JP and Pearce N. Incidence rates in dynamic populations. Int J Epidemiol 2012; 41: 1472-1479.

12. Institut d'Estadística de Catalunya (Idescat), Generalitat de Catalunya. http://www.idescat.cat/ pub/?id=pmh (Updated 1 January 2017, accessed 16 June 2017).

13. Bukhari W, Prain KM, Waters P, et al. Incidence and prevalence of NMOSD in Australia and New Zealand. J Neurol Neurosurg Psychiatry 2017; 88: 632-638.

14. Mayr WT, Pittock SJ, McClelland RL, et al. Incidence and prevalence of multiple sclerosis in Olmsted County, Minnesota, 1985-2000. Neurology 2003; 61: 1373-1377.

15. Rees F, Doherty M, Grainge Davenport G, et al. The incidence and prevalence of systemic lupus erythematosus in the UK, 1999-2012. Ann Rheum Dis 2016; 75: 136-141.

16. Chitnis T, Ness J, Krupp L, et al. Clinical features of neuromyelitis optica in children: US Network of Pediatric MS centers report. Neurology 2016; 86: 245-252.

17. Quek AM, McKeon A, Lennon VA, et al. Effects of age and sex on aquaporin-4 autoimmunity. Arch Neurol 2012; 69: 1039-1043.

18. Jiao Y, Fryer JP, Lennon VA, et al. Updated estimate of AQP4-IgG serostatus and disability outcome in neuromyelitis optica. Neurology 2013; 81: 11971204.

19. Farré N, Vela E, Clèries M, et al. Medical resource use and expenditure in patients with chronic heart failure: A population-based analysis of 88,195 patients. Eur J Heart Fail 2016; 18: 1132-1140.
Visit SAGE journals online journals.sagepub.com/ home/msj

(SAGEjournals 